Rev. Int. Contam. Ambie. 33 (2) 183-197, 2017

DOI: 10.20937/RICA.2017.33.02.01

\title{
FORMALDEHÍDO EN VIVIENDAS URBANAS EN SALVADOR, BAHÍA - BRASIL
}

\author{
Norma Irene SOZA PINEDA ${ }^{1 *}$, Pedro Reginaldo DOS SANTOS PRATA ${ }^{2}$, Maria Regina \\ ALVES CARDOSO ${ }^{3}$, Gisele OLÍMPIO DA ROCHA ${ }^{4}$, Leila Denise ALVES FERREIRA AMORÍN ${ }^{5}$, \\ Renata DE MIRANDA ESQUIVEL ${ }^{6}$, Fabiana SILVA DE CARVALHO ${ }^{4}$ y Mauricio LIMA BARRETO ${ }^{7}$
}

${ }^{1}$ Universidade do Estado da Bahia. Rua Silveira Martins, 2555, Cabula, Salvador, Brasil, CEP 41.150-000

${ }^{2}$ Instituto de Saúde Coletiva, Universidade Federal da Bahia. Rua Basílio da Gama, sin número, Canela, Salvador, Brasil, CEP 40110-040

${ }^{3}$ Universidade de São Paulo, Avenida Dr. Arnaldo 715, São Paulo, CEP 01246-904

${ }^{4}$ Instituto de Química Analítica, Universidade Federal da Bahia. Rua Barão de Jeremoabo 147, Campus Universitário de Ondina - Salvador, Brasil, CEP 40170-115

${ }^{5}$ Departamento de Estatística, Instituto de Matemática, Universidade Federal da Bahia. Rua Ademar de Barros, sin número, Campus Universitário de Ondina, Salvador, Brasil, CEP 40170-115

${ }^{6}$ Universidade Salvador. Rua Dr. Jose Peroba 251, Edifício Civil Empresarial, sala 701, Stiep, Salvador, Brasil, CEP 41770-235

${ }^{7}$ Universidade Federal da Bahia. Rua Basílio da Gama, Canela, Salvador, Brasil, CEP 40110-040

*Autor para correspondencia: normasoza@hotmail.com

(Recibido mayo 2015; aceptado agosto 2016)

Palabras clave: aldehídos, contaminación del aire en interiores, exposición a riesgos ambientales

\section{RESUMEN}

El formaldehído es un contaminante común en los espacios interiores. El objetivo de este estudio fue identificar la concentración de formaldehído en viviendas urbanas y la posible relación de las fuentes potenciales intramuros con dicha concentración. Se utilizó un método transversal anidado en un estudio longitudinal que investiga factores asociados con el asma y la alergia en niños escolares en Salvador. Entre febrero de 2007 y julio de 2008 se visitaron las viviendas de 1168 niños (4 - 13 años) para recolectar información sobre las características de las casas. Para la determinación de formaldehído gaseoso se utilizó un procedimiento fluorimétrico con el reactivo Fluoral P. Se evaluaron los indicadores de ventilación, fuentes de formaldehído y exposición al humo del cigarro según los niveles de formaldehído a través de una regresión logística, en la que se calculó la razón de productos cruzados (RPC) con un intervalo de confianza (IC) de $95 \%$. La concentración de formaldehído en general fue baja (media $0.80 \mu \mathrm{g} / \mathrm{m}^{3}$ ). Un mayor número de fuentes potencialmente emisoras de ese compuesto (RPC 1.30; $95 \%$ IC 1.03-1.65), así como las mejores condiciones de ventilación (RPC 1.35; 95 \% IC 1.07-1.70), estuvieron asociadas con una mayor concentración de formaldehído. Mientras que en las viviendas donde habitaban fumadores, la concentración de formaldehído fue menor (RPC 0.69; 95 \% IC $0.52-0.90)$. El nivel de concentración del formaldehído en las viviendas estudiadas en Salvador fue inferior a los niveles máximos aceptables nacional e internacionalmente para ambientes interiores. Las características de las viviendas influyeron en la concentración de dicho compuesto. El método de muestreo ambiental utilizado permitió la determinación del formaldehído con suficiente confiabilidad.

Key words: aldehydes, indoor air contamination, exposure to environmental risks 


\begin{abstract}
Formaldehyde is a common contaminant in indoor environments. The objective of the present study was to identify formaldehyde concentrations in urban households and to investigate a possible association between the formaldehyde concentration and potential indoor sources. This is a cross-sectional study nested within SCAALA, a large longitudinal study conducted to investigate factors associated with asthma and allergy in schoolchildren in Salvador. The homes of 1168 children from 4 to 13 years old were visited between February 2007 and July 2008 to collect data on the household characteristics. Fluorometric determination of gaseous formaldehyde was conducted using the Fluoral-P reagent. Logistic regression with calculation of odds ratios and their respective $95 \%$ confidence intervals $(95 \% \mathrm{CI})$, was used to evaluate ventilation, sources of formaldehyde, and exposure to cigarette smoke according to formaldehyde concentrations. Formaldehyde concentrations were low (a mean of $0.80 \mu / \mathrm{m} 3$ ). A higher number of potential sources of formaldehyde emission (OR: 1.30; $95 \%$ CI: $1.03-1.65$ ) and better ventilation (OR: 1.35 ; $95 \%$ CI: $1.07-1.70$ ) were factors associated with higher concentrations. Formaldehyde concentrations were lower in homes where there were smokers, (OR: 0.69; $95 \%$ CI: $0.52-0.90)$. Indoor formaldehyde concentrations in the homes evaluated in Salvador were below the maximum allowable concentrations determined nationally and internationally. The characteristics of the homes played a role in formaldehyde levels. The environmental sampling method used to determine formaldehyde concentrations was sufficiently accurate.
\end{abstract}

\section{INTRODUCCIÓN}

El formaldehído forma parte de un grupo de químicos orgánicos llamados compuestos orgánicos volátiles (COV). A temperatura ambiente es un gas incoloro, de olor penetrante, muy reactivo y de alta solubilidad en agua y en ésteres. Su presentación comercial más conocida es la formalina, pero también se fabrica en forma de resinas que sirven de aditivos en la confección de productos de madera y materiales de construcción. Además, se usa como materia prima en varios procesos industriales donde actúa como conservador, desinfectante o biocida. (Salthammer et al. 2010).

La concentración de formaldehído tiende a ser mayor en los ambientes interiores que en los exteriores (Zhang et al. 1994, Báez et al. 2003, Gioda y Aquino 2003). Las fuentes intramuros incluyen principalmente las emisiones del material con el que se elaboran paredes y muebles (Dally et al. 1981 y Gilbert et al. 2006), materiales de acabado y decoración a base de madera (Zhao et al. 2004), tableros de fibra de madera conglomerada (Kelly et al. 1999), emisiones gaseosas del alfombrado (Weislander et al. 1997 y Ohura et al. 2006), disolventes y pinturas (Norback et al. 1995, Gilbert et al. 2006), productos de limpieza y pesticidas (Wolcoff et al. 1998), cosméticos y productos de higiene personal (Kelly et al. 1999), humo del cigarro (Godish 1989), combustión de la madera (De Andrade et al. 2002) y del gas natural (Vaizoglu et al. 2003 y Corrêa y Arbilla 2005).

En áreas distantes de las zonas urbanas (costas, océanos y montañas) se han reportado concentraciones entre $0.1 \mu \mathrm{g}$ y $2.71 \mu \mathrm{g}$, y en el aire exterior de áreas urbanas contaminadas por el tránsito de vehículos entre $1.59 \mu \mathrm{g}$ y $83.39 \mu \mathrm{g}$ (De Andrade et al. 2002). En viviendas de países industrializados se han reportado concentraciones medias de formaldehído entre 15.41 $\mu \mathrm{g} / \mathrm{m}^{3}$ y $75 \mu \mathrm{g} / \mathrm{m}^{3}$ en el área de la cocina (Zhang et al. 1994, Vaizoglu et al. 2003), entre $12.4 \mu \mathrm{g} / \mathrm{m}^{3}$ y $134 \mu \mathrm{g} / \mathrm{m}^{3}$ en la sala (Lee et al. 1999, Vaizoglu et al. 2003, Gilbert et al. 2006, Ohura et al. 2006, Park e Ikeda 2006, Sax et al. 2006, Marchand et al. 2008, Guo et al. 2009, Heroux et al. 2010, Villanueva et al. 2015), entre $22 \mu \mathrm{g} / \mathrm{m}^{3}$ y $33 \mu \mathrm{g} / \mathrm{m}^{3}$ en el cuarto o dormitorio (Weislander et al. 1997, Garret et al. 1999, Gonzalez-Flesca et al. 1999, Lee et al. 1999, Runchev et al. 2002, Venn et al. 2003, Marchand et al. 2008), y entre $32 \mu \mathrm{g} / \mathrm{m}^{3}$ y $130 \mu \mathrm{g} / \mathrm{m}^{3}$ como concentración media de varias muestras dentro de una misma vivienda (Dingle y Frankling 2002, Zhao et al. 2004, Gilbert et al. 2005).

En Brasil, han sido reportadas concentraciones entre $0.0 \mu \mathrm{g} / \mathrm{m}^{3}$ y $188.0 \mu \mathrm{g} / \mathrm{m}^{3}$ en interiores y entre $0.3 \mu \mathrm{g} / \mathrm{m}^{3}$ y $30.3 \mu \mathrm{g} / \mathrm{m}^{3}$ en exteriores (Gioda y Aquino 2003). Esos estudios han incluido centros de trabajo como restaurantes, oficinas y universidades. Las muestras en el aire exterior fueron colectadas en patios, áreas abiertas o ventanas de los edificios o 
casas, a una altura entre $1.5 \mathrm{~m}$ del suelo y hasta el $25^{\circ}$ piso de un edificio. La concentración en viviendas fue de $40 \mu \mathrm{g} / \mathrm{m}^{3}$ en Río de Janeiro (Gioda y Aquino 2003) y de $29 \mu \mathrm{g} / \mathrm{m}^{3}$ en San Pablo (Pires y Carvalho 1999).

En general las personas pasan más del $80 \%$ de su tiempo dentro de inmuebles (vivienda, escuela, centro de trabajo, lugares públicos) y $6 \%$ dentro de vehículos (Kepleis et al. 2001). El estudio de la concentración de formaldehído en interiores es relevante debido a que la exposición a ese compuesto ha sido asociada con problemas respiratorios en niños y adultos (IOM 2004).

En Brasil hay pocos estudios que relacionan las concentraciones de formaldehído gaseoso con la calidad del aire en las viviendas. Este estudio pretende identificar los niveles de formaldehído habituales en las viviendas de Salvador, lo que podría contribuir a definir los niveles máximos permitidos que garanticen una buena calidad del aire y que en consecuencia, se disminuya la exposición humana a este compuesto. Por lo anterior, el objetivo del trabajo fue identificar la concentración de formaldehído y su relación con fuentes potenciales en el interior de viviendas en Salvador, Bahía, Brasil.

\section{MATERIALES Y MÉTODOS}

Se utilizó un método transversal anidado en un estudio longitudinal, llevado a cabo entre febrero de 2007 a julio de 2008. El sitio de estudio fue la ciudad de Salvador, capital del estado de la Bahía, localizada en el noreste de Brasil, con una población estimada de 2714018 hab en 2007 (IBGE 2000). Salvador posee un clima tropical con temperatura $\left(25^{\circ} \mathrm{C}\right)$ y humedad ambiental $(81 \%)$ relativamente constantes y lluvia abundante durante casi todo el año.

Este trabajo surge del programa de investigación "Cambios sociales, asma y alergia en Latinoamérica" (SCAALA, por sus siglas en inglés), que fue diseñado para estudiar factores de riesgo para asma y otras enfermedades alérgicas en una generación de niños escolares en Salvador, Bahía. La población del programa SCAALA fue seleccionada en un estudio anterior que tuvo por objetivo evaluar el impacto de saneamiento urbano sobre la salud de los niños. En 1997, 2001 y 2003, tres diferentes generaciones de niños de 0 a 3 años fueron seleccionadas a través de un muestreo aleatorio en 24 de las 30 micro-áreas de la zona urbana de la ciudad de Salvador. Dicho muestreo fue representativo de las zonas más marginadas de la población residente en Salvador (Barreto et al. 2006).
En 2005 se obtuvo información sobre la prevalencia de asma y factores de riesgo asociados a ella y a alergias. Un total de 1445 niños de ambos sexos (4-13 años) integraron esta generación. En este estudio participaron 1168 niños $(80.8 \%)$ y sus respectivos padres o tutores. El protocolo del estudio fue aprobado por la comisión de ética de la Universidad Federal de la Bahía y el Ministerio de Salud de Brasil. Se solicitó consentimiento escrito a los responsables para la participación del niño en el estudio.

Se utilizó un cuestionario estructurado, mismo que fue entregado al responsable del niño. Por simple inspección se constataron las características del ambiente de la vivienda y de los muebles. Además, se comprobó el uso de los productos de higiene personal y de limpieza al solicitar los envases vacíos o en uso cuando estuvieron disponibles. Previamente a los cuestionarios se realizó una prueba piloto con madres que no pertenecían a la muestra, para evaluar la comprensión de las preguntas y realizar los ajustes necesarios. Las encuestadoras fueron entrenadas para la recolección de los datos. Asimismo, se realizaron entrevistas de verificación en $10 \%$ de las viviendas de la muestra.

La variable dependiente fue la concentración del formaldehído expresada en $\mu \mathrm{g} / \mathrm{m}^{3}$. Las variables independientes fueron agrupadas en: 1) características del ambiente de la vivienda (presencia de alfombrados, plantas o animales, uso de productos de limpieza, tipo de material de los muebles, como madera maciza, conglomerado de fibra de madera, madera contrachapada, metal, cuero, tela o plástico), 2) características físicas de las viviendas (tipo y antigüedad, área geográfica, última pintura, última remodelación), 3) características de la ventilación (número de ventanas por cuarto, área de ventilación, periodo en que las ventanas permanecían abiertas, percepción de olores fuertes dentro de la vivienda) y 4) estilo de vida (hábito de fumar).

\section{Muestreo ambiental}

En este estudio se utilizó un método fluorimétrico, que es más sensible y específico para la determinación de formaldehído gaseoso. Se basa en la reacción entre el formaldehído y el reactivo Fluoral P, lo que produce el compuesto fluorescente 3.5-diacetil-1-4-dihidrolutidina (DDL), mismo que al ser excitado a $410 \mathrm{~nm}$, emite fluorescencia a $510 \mathrm{~nm}$. El reactivo Fluoral P, fue preparado con la reacción de $0.3 \mathrm{~mL}$ de ácido acético, $0.3 \mathrm{~mL}$ de acetilacetona bidestilada y $15.4 \mathrm{~g}$ de acetato de amonio, con agua desmineralizada para un volumen de $100 \mathrm{~mL}$ (De Andrade et al. 1995). Posteriormente fue validado por comparación (Pinheiro et al. 2004) con el mé- 
todo de referencia (Levin et al. 1985), que utiliza cartuchos C18 impregnados con solución ácida de 2.4-dinitrofenilhidracina. El análisis es realizado por cromatografía líquida de alta eficiencia (CLAE). Entre las principales ventajas del método, se encuentra que no hay interferencia con la concentración de acetaldehído, incluso en concentraciones 1000 veces mayores que las de formaldehído. Además, presenta bajos niveles de blanco, bajo límite de detección, buena precisión y requiere de poca manipulación en el tratamiento de las muestras (Pinheiro et al. 2004).

Se realizó un muestreo piloto en 50 viviendas pertenecientes a la muestra para comparar las concentraciones entre la sala y el cuarto donde el niño dormía. Por simple comparación se observó que las concentraciones eran muy similares en los dos lugares, por esa razón se escogió para el muestreo el cuarto del niño, debido al mayor tiempo de permanencia dentro de él ( $8 \mathrm{~h} /$ día). Se utilizó un filtro como blanco para todos los que fueron muestreados por día. En la habitación, el filtro fue colocado sobre algún mueble distante de fuentes de ventilación o radiación, a $1.5 \mathrm{~m}$ del nivel del piso, por $120 \mathrm{~h}$, periodo considerado satisfactorio para cuantificar formaldehído. Las muestras fueron analizadas por dos personas calificadas, con reactivos de grado cromatográfico (Merck) en un laboratorio específicamente para esa actividad.

\section{Análisis}

Los cuestionarios fueron revisados para descartar posibles errores durante la toma de información. Posteriormente, se realizaron análisis estadísticos con el paquete "SPSS" v.13, después de un análisis de consistencia.

Se calcularon coeficientes descriptivos para variables cuantitativas y de distribución de frecuencia para variables cualitativas. Fueron utilizadas las pruebas de Wilcoxon-Mann-Whitney y de Kruskal-Wallis para comparar la concentración de formaldehído entre los grupos según las características de la ventilación, la presencia de fuentes potenciales de formaldehído y la exposición al humo del cigarro. Con el modelo de tendencia se evaluó la asociación entre niveles de formaldehído (diferenciadas por la mediana) con la ventilación y las fuentes potenciales de formaldehído que fueron definidas según un análisis multivariado en categorías con escala ordinal. En el análisis multivariado se utilizó el análisis factorial de correspondencia múltiple (AFC) y el análisis de detección de conglomerados (cluster, por su término en inglés) a través del paquete "SPAD" v.3.5.

Para evaluar la asociación entre las características de la vivienda y las fuentes potenciales de formaldehído con la concentración en el cuarto, se seleccionaron variables que según la literatura se han asociado con la concentración de formaldehído doméstico (Dally et al. 1981, Godish 1989, Weislander et al. 1997, Wolcoff et al. 1998, Zhao et al. 2004, Gilbert et al. 2005, Ohura et al. 2006, Heroux et al. 2010).

\section{RESULTADOS}

La mayoría de las casas tenían cinco años de antigüedad, con paredes de concreto y habían sido pintadas o remodeladas hacía más de seis meses. En $74 \%$ de ellas había más de una ventana, las que en su mayoría permanecían abiertas por más de la mitad del día. En más de la mitad de las casas fue posible percibir algún olor fuerte en la sala y en el cuarto donde el niño dormía y en $85 \%$ se utilizaban más de cuatro productos de limpieza. En $60 \%$ de las casas en el cuarto donde dormía el niño, había al menos una ventana y no se acostumbraba la permanencia de animales, plantas o alfombrado en ese espacio (Cuadro I).

El tamaño promedio de las casas fue $42.35 \mathrm{~m}^{2}$ $\left(5.18 \mathrm{~m}^{2}-148.03 \mathrm{~m}^{2}\right)$ con un promedio de 2.82 (0 - 13) ventanas y una área total de ventilación que osciló entre $0.54 \mathrm{~m}^{2}$ y $17.53 \mathrm{~m}^{2}$, siendo mayor en aquellas casas que contaban con salas de estar abiertas. En promedio había cuatro cuartos, dos de los cuales eran destinados para dormir. El tamaño promedio del cuarto donde el niño dormía fue $8.43 \mathrm{~m}^{2}$ $\left(1.48 \mathrm{~m}^{2}-32.11 \mathrm{~m}^{2}\right)$ y en su mayoría no había ventanas (promedio 0.65). Por lo general, el niño compartía el cuarto con los padres o hermanos y en pocos casos dormía en la sala. En todas las casas la puerta del cuarto era interior (datos no mostrados).

La concentración media de formaldehído en el cuarto del niño fue de $0.80 \mu \mathrm{g} / \mathrm{m}^{3}\left(0.00 \mu \mathrm{g} / \mathrm{m}^{3}-11.1 \mu \mathrm{g} / \mathrm{m}^{3}\right)$. Fueron excluidas tres viviendas por presentar concentraciones atípicas en relación con el resto $\left(19.3 \mu \mathrm{g} / \mathrm{m}^{3}, 23.1 \mu \mathrm{g} / \mathrm{m}^{3}, 31 \mu \mathrm{g} / \mathrm{m}^{3}\right)$, no identificándose la causa de esas diferencias.

En el cuadro II, se puede observar que la concentración de formaldehído fue mayor en viviendas remodeladas $\left(0.42 \mu \mathrm{g} / \mathrm{m}^{3} \mathrm{p}=0.014\right)$ o pintadas en los últimos seis meses $\left(0.42 \mu \mathrm{g} / \mathrm{m}^{3} \mathrm{p}=0.019\right)$, que poseían más de una ventana $\left(0.39 \mu \mathrm{g} / \mathrm{m}^{3} \mathrm{p}=0.028\right)$ y tenían muebles de varios tipos de material $\left(0.42 \mu \mathrm{g} / \mathrm{m}^{3}\right.$ $\mathrm{p}=0.041)$, mismas en las que no se percibieron olores fuertes en la sala $\left(0.42 \mu \mathrm{g} / \mathrm{m}^{3} \mathrm{p}=0.024\right)$ y usaban más de siete productos de limpieza $(0.43 \mu \mathrm{g} /$ $\left.\mathrm{m}^{3} \mathrm{p}=0.034\right)$. Asimismo en las casas que tenían más de una ventana $\left(0.44 \mu \mathrm{g} / \mathrm{m}^{3} \mathrm{p}=0.005\right)$ en el cuarto 
CUADRO I. CARACTERÍSTICAS DE LAS FUENTES POTENCIALES DE FORMALDEHÍDO Y DE LA VENTILACIÓN EN VIVENDAS DE NIÑOS DE EDAD ESCOLAR RESIDENTES EN LA CIUDAD DE SALVADOR - BAHÍA, BRASIL, 2007

\begin{tabular}{|c|c|c|}
\hline Variables & $\mathrm{N}$ & $\%$ \\
\hline \multicolumn{3}{|l|}{ Edad de la vivienda $(\mathrm{n}=1165)$} \\
\hline$\leq 5$ años. & 145 & 12.44 \\
\hline$>5$ años. & 1020 & 87.56 \\
\hline \multicolumn{3}{|c|}{ Material de las paredes de la vivienda $(\mathrm{n}=1165)$} \\
\hline Concreto & 1154 & 99.05 \\
\hline Otros materiales & 11 & 0.95 \\
\hline \multicolumn{3}{|l|}{ Última pintura en la vivienda $(\mathrm{n}=1165)$} \\
\hline Nunca/ no sabe & 140 & 12.01 \\
\hline Menos de 6 meses & 340 & 29.19 \\
\hline Más de 6 meses & 685 & 58.80 \\
\hline \multicolumn{3}{|c|}{ Reedificación en la vivienda (piso o pared) $(\mathrm{n}=1165)$} \\
\hline Menos de 6 meses & 390 & 33.48 \\
\hline Más de 6 meses & 775 & 66.52 \\
\hline \multicolumn{3}{|c|}{ Material de los muebles (madera, madera conglomerada) $(\mathrm{n}=1165)$} \\
\hline Posee máximo uno de los materiales & 194 & 16.67 \\
\hline Posee dos tipos de materiales & 587 & 50.36 \\
\hline Posee los tres tipos de materiales & 384 & 32.97 \\
\hline \multicolumn{3}{|c|}{ Características del lugar donde el niño dormía $(\mathrm{n}=1165)$} \\
\hline Ausencia de animal, planta o alfombra & 766 & 65.35 \\
\hline Presencia de animal, planta o alfombra & 399 & 34.25 \\
\hline \multicolumn{3}{|l|}{ Uso de productos de limpieza $(n=1165)$} \\
\hline Hasta 3 tipos diferentes & 172 & 14.73 \\
\hline 4 a 6 & 930 & 79.89 \\
\hline$\geq 7$ & 63 & 5.38 \\
\hline \multicolumn{3}{|c|}{ Número de ventanas en la vivienda $(n=1165)$} \\
\hline Hasta una ventana & 294 & 26.0 \\
\hline Más de una ventana & 871 & 74.0 \\
\hline \multicolumn{3}{|c|}{ Período en que las ventanas permanecían abiertas $(\mathrm{n}=1165)$} \\
\hline Más de la mitad del día & 990 & 84.8 \\
\hline Menos de la mitad del día & 76 & 6.5 \\
\hline No tiene ventanas & 102 & 8.7 \\
\hline \multicolumn{3}{|c|}{ Número de ventanas en el cuarto donde el niño dormía $(\mathrm{n}=1165)$} \\
\hline Ninguna ventana & 441 & 37.9 \\
\hline Una ventana & 697 & 59.8 \\
\hline Más de uma & 27 & 2.3 \\
\hline \multicolumn{3}{|l|}{ Olores fuertes en la sala $(n=1165)$} \\
\hline Ninguno & 532 & 45.7 \\
\hline Por lo menos uno & 633 & 54.3 \\
\hline \multicolumn{3}{|l|}{ Olores fuertes en el cuarto $(n=1165)$} \\
\hline Ninguno & 557 & 47.8 \\
\hline Por lo menos uno & 608 & 52.2 \\
\hline
\end{tabular}


CUADRO II. COMPARACIÓN DE LOS NIVELES DE FORMALDEHÍDO ENTRE LAS CATEGORÍAS DE LAS VARIABLES RELACIONADAS A LAS CONDICIONES DE VENTILACIÓN Y A LAS FUENTES POTENCIALES DE FORMALDEHÍDO EN LAS VIVIENDAS DE SALVADOR, BRASIL, 2007

\begin{tabular}{|c|c|c|c|}
\hline Características de la vivienda & $\mathrm{N}$ & Mediana (Q1-Q3)*** & p-valor \\
\hline \multicolumn{4}{|l|}{ VENTILACIÓN } \\
\hline \multicolumn{4}{|c|}{ Número de ventanas en la vivienda $(\mathrm{n}=1165)^{*}$} \\
\hline Hasta una ventana & 294 & $0.326(0.145-0.634)$ & \multirow[b]{2}{*}{0.028} \\
\hline Más de una ventana & 871 & $0.390(0.161-0.838)$ & \\
\hline \multicolumn{4}{|c|}{ Período en que las ventanas permanecían abiertas $(\mathrm{n}=1165)^{* *}$} \\
\hline Más de la mitad del día & 990 & $0.377(0.157-0.808)$ & \multirow[t]{3}{*}{0.946} \\
\hline Menos de la mitad del día & 76 & $0.343(0.168-0.843)$ & \\
\hline No había ventanas & 102 & $0.322(0.136-0.833)$ & \\
\hline \multicolumn{4}{|c|}{$\mathrm{N}^{\circ}$ de ventanas en el lugar donde el niño dormía $(\mathrm{n}=1165)^{* *}$} \\
\hline Ninguna ventana & 441 & $0.317(0.133-0.670)$ & \multirow[b]{3}{*}{0.005} \\
\hline Una ventana & 697 & $0.417(0.173-0.876)$ & \\
\hline Más de una ventana & 27 & $0.442(0.161-0.692)$ & \\
\hline \multicolumn{4}{|l|}{ Olores fuertes en la sala $(n=1165)^{*}$} \\
\hline Ninguno & 532 & $0.422(0.194-0.877)$ & \multirow[b]{2}{*}{0.005} \\
\hline Por lo menos uno & 633 & $0.325(0.129-0.727)$ & \\
\hline \multicolumn{4}{|c|}{ Olores fuertes en el lugar donde el niño dormía $(n=1165)^{*}$} \\
\hline Ninguno & 557 & $0.421(0.177-0.866)$ & \multirow[b]{2}{*}{0.024} \\
\hline Por lo menos uno & 608 & $0.334(0.140-0.757)$ & \\
\hline \multicolumn{4}{|l|}{ FUENTES DE FORMALDEHÍDO } \\
\hline \multicolumn{4}{|c|}{ Tipos de materiales de los muebles de la casa $(\mathrm{n}=1165)^{* *}$} \\
\hline Posee 1 tipo de material & 194 & $0.294(0.111-0582)$ & \multirow{3}{*}{0.041} \\
\hline Posee 2 tipos de materiales & 587 & $0.372(0.144-0.800)$ & \\
\hline Posee 3 tipos de materiales & 384 & $0.425(0.188-1.025)$ & \\
\hline \multicolumn{4}{|c|}{ Característica del lugar donde el niño dormía $(\mathrm{n}=1165)^{*}$} \\
\hline Ausencia de animal, planta, o alfombra & 766 & $0.386(0.170-0.819)$ & \multirow[t]{2}{*}{0.060} \\
\hline Presencia de animal, planta, o alfombra & 399 & $0.323(0.131-0.777)$ & \\
\hline \multicolumn{4}{|l|}{ Edad de la vivienda $(\mathrm{n}=1165)^{*}$} \\
\hline$<5$ anos & 145 & $0.428(0.195-1.012)$ & \multirow[b]{2}{*}{0.105} \\
\hline$>5$ anos & 1020 & $0.801(0.364-2.003)$ & \\
\hline \multicolumn{4}{|l|}{ Última reforma en la vivienda $(\mathrm{n}=1165)^{*}$} \\
\hline Menos de 6 meses & 390 & $0.423(0.181-0.840)$ & \multirow[b]{2}{*}{0.014} \\
\hline Más de seis meses & 775 & $0.339(0.140-0.789)$ & \\
\hline \multicolumn{4}{|l|}{ Última pintura en la vivienda $(\mathrm{n}=1165)^{* *}$} \\
\hline Nunca/no sabe & 140 & $0.310(0.112-0.623)$ & \multirow{3}{*}{0.019} \\
\hline Menos de 6 meses & 340 & $0.427(0.194-0,859)$ & \\
\hline Más de 6 meses & 685 & $0.349(0.147-0,803)$ & \\
\hline \multicolumn{4}{|c|}{ Material de las paredes de la vivienda $(\mathrm{n}=1165)^{*}$} \\
\hline Concreto & 1154 & $0.373(0.158-0.808)$ & \multirow[b]{2}{*}{0.247} \\
\hline Otros materiales & 11 & $0.147(0.088-0.513)$ & \\
\hline
\end{tabular}

*p-valor de la prueba de Wilcoxon-Mann-Whitney, ** p-valor de la prueba de Kruskal-Wallis, *** $\mu \mathrm{g} / \mathrm{m}^{3}$, números en negritas denotan diferencias significativa. Q1-Q3 = cuartil 1- cuartil 3 
CUADRO II. COMPARACIÓN DE LOS NIVELES DE FORMALDEHÍDO ENTRE LAS CATEGORÍAS DE LAS VARIABLES RELACIONADAS A LAS CONDICIONES DE VENTILACIÓN Y A LAS FUENTES POTENCIALES DE FORMALDEHÍDO EN LAS VIVIENDAS DE SALVADOR, BRASIL, 2007

\begin{tabular}{|c|c|c|c|}
\hline Características de la vivienda & $\mathrm{N}$ & Mediana $(\mathrm{Q} 1-\mathrm{Q} 3)^{* * *}$ & p-valor \\
\hline \multicolumn{4}{|c|}{ Uso de productos de limpieza $(\mathrm{n}=1165)^{* *}$} \\
\hline Hasta 3 tipos & 172 & $0.292(0.116-0.629)$ & \multirow{3}{*}{0.034} \\
\hline 4-6 tipos & 933 & $0.377(0.161-0.820)$ & \\
\hline Más de 7 tipos & 63 & $0.433(0.224-1.365)$ & \\
\hline \multicolumn{4}{|c|}{ Exposición al humo de cigarro $(\mathrm{n}=1165)^{*}$} \\
\hline Sin exposición en el domicilio & 886 & $0.396(0.177-0.875)$ & \multirow[b]{2}{*}{0.000} \\
\hline Con exposición en el domicilio & 279 & $0.291(0.114-0.616)$ & \\
\hline
\end{tabular}

*p-valor de la prueba de Wilcoxon-Mann-Whitney, ** p-valor de la prueba de Kruskal-Wallis, *** $\mu \mathrm{g} / \mathrm{m}^{3}$, números en negritas denotan diferencias significativa. Q1-Q3 = cuartil 1- cuartil 3

donde el niño dormía, se habían percibido olores fuertes durante la toma de información $\left(0.42 \mu \mathrm{g} / \mathrm{m}^{3}\right.$ $\mathrm{p}=0.005)$. La concentración de formaldehído también fue mayor donde el niño no estaba expuesto al humo del cigarro $\left(0.39 \mu \mathrm{g} / \mathrm{m}^{3} \mathrm{p}<0.001\right)$.

Para evaluar las relaciones entre las variables, éstas se agruparon en conjuntos de menor dimensión con el AFC. Se observó una asociación entre las variables que revelaban mejores condiciones de ventilación, inadecuadas condiciones de ventilación y relacionadas a las fuentes potenciales de formaldehído (datos no mostrados).

Se detectaron conglomerados con el objetivo de constituir grupos en relación con las condiciones de ventilación: "poca ventilación", "ventilación moderada" y "buena ventilación". Así como con las fuentes de formaldehído: "pocas fuentes", "moderado número de fuentes" y "múltiples fuentes" (datos no mostrados).

Se verificó que la proporción de viviendas con mayor concentración de formaldehído aumentaba a medida que mejoraba la ventilación del ambiente. Así, $54.5 \%$ de las viviendas con buena ventilación $\left(>0.44 \mu \mathrm{g} / \mathrm{m}^{3} \mathrm{p}=0.026\right)$ y $53.8 \%$ de las clasificadas con múltiples fuentes de formaldehído $\left(>0.44 \mu \mathrm{g} / \mathrm{m}^{3}\right.$ $\mathrm{p}=0.019)$ presentaron concentraciones por encima de la mediana (Cuadro III).

En el cuadro IV se observa que viviendas con un número moderado de fuentes de formaldehído tuvieron un aumento de $36 \%$ en la probabilidad de presentar concentraciones por encima de la mediana, comparadas con aquellas con menos fuentes (RPC $=1.36 ; 95 \%$ IC: $1.05-1.75)$. Las viviendas con presencia de fumadores tuvieron una probabilidad $30 \%$ menor de presentar alta concentración de formaldehído en comparación con aquellas donde no había fumadores (RPC $=0.70$; $95 \%$ IC: 0.53-0.92).

En los cuadros III y IV se comprobó que la concentración de formaldehído fue similar en los grupos de "poca" y "moderada" ventilación, así como en los grupos de "moderadas" y "múltiples" fuentes de formaldehído. Así, se juntaron ambas categorías y

CUADRO III. PROPORCIÓN DE VIVIENDAS CON CONCENTRACIÓN DE FORMALDEHÍDO SUPERIOR A LA MEDIANA SEGÚN GRUPOS DEFINIDOS EN ANÁLISIS MULTIVARIADO

\begin{tabular}{|c|c|c|}
\hline $\begin{array}{l}\text { Indicadores del análisis de } \\
\text { conglomerados }\end{array}$ & $\begin{array}{l}\text { Proporción de viviendas con mayor } \\
\text { concentración de formaldehido (\%) }\end{array}$ & p-valor* \\
\hline \multicolumn{3}{|l|}{ Grupos según ventilación } \\
\hline Poca ventilación $(\mathrm{n}=504)$ & 46.6 & \\
\hline Moderada ventilación $(\mathrm{n}=483)$ & 47.4 & 0.026 \\
\hline Buena ventilación $(\mathrm{n}=178)$ & 54.5 & \\
\hline \multicolumn{3}{|l|}{ Grupos según fuentes de formaldehido } \\
\hline Pocas fuentes $(n=629)$ & 46.6 & \\
\hline Moderado número de fuentes $(\mathrm{n}=391)$ & 54.7 & 0.019 \\
\hline Múltiplas fuentes $(\mathrm{n}=145)$ & 53.8 & \\
\hline
\end{tabular}

* prueba de tendencia, números en negritas denotan diferencias significativas 
CUADRO IV. CÁLCULO DE LA RAZÓN DE PRODUCTOS CRUZADOS (RPC) Y SUS INTERVALOS DE $95 \%$ DE CONFIANZA SEGÚN MODELO DE REGRESIÓN LOGÍSTICA

\begin{tabular}{lcc}
\hline Variables & RPC & $95 \%$ IC \\
\hline $\begin{array}{l}\text { Moderada ventilación } \\
\quad(\text { Ref = poca ventilación) }\end{array}$ & 1.0278 & $(0.73 ; 1.45)$ \\
$\begin{array}{l}\text { Buena ventilación } \\
\quad \text { (Ref = poca ventilación) }\end{array}$ & 1.3121 & $(0.93 ; 1.86)$ \\
$\begin{array}{l}\text { Moderado número de fuentes de } \\
\text { formaldehído } \\
\quad \text { (Ref = pocas fuentes) }\end{array}$ & $\mathbf{1 . 3 5 9 8}$ & $(1.05 ; 1.75)$ \\
$\begin{array}{l}\text { Múltiples fuentes de formaldehído } \\
\quad \text { (Ref = pocas fuentes) }\end{array}$ & 1.3386 & $(0.93 ; 1.93)$ \\
$\begin{array}{l}\text { Presencia de fumadores en la } \\
\text { vivienda } \\
\quad(\text { Ref }=\text { ausencia de fumadores) }\end{array}$ & $\mathbf{0 . 6 9 6 3}$ & $(0.53 ; 0.92)$ \\
\hline
\end{tabular}

Ref = grupo de referencia, $\mathrm{IC}=$ Intervalo de confianza, números en negritas denotan diferencias significativas

el análisis fue realizado nuevamente considerándose sólo dos grupos de viviendas, tanto en relación con la ventilación ("poca-moderada ventilación" y "buena ventilación") como con las fuentes de formaldehído ("pocas" y "moderadas-múltiples fuentes"). En el cuadro $\mathbf{V}$ hubo un aumento en la concentración de formaldehído en ambientes con "buena ventilación" $(\mathrm{p}=0.015)$ y con "moderadas a múltiples" fuentes de formaldehído $(\mathrm{p}=0.007)$.

La probabilidad de presentar niveles más altos de formaldehído fue $30 \%$ mayor en viviendas con buena ventilación que en las que tenían poca o moderada ventilación $(\mathrm{RPC}=1.30$; 95 \% IC: $1.03-1.65)$. La probabilidad de presentar niveles de formaldehído por encima de la mediana fue $35 \%$ mayor en viviendas con moderadas a múltiples fuentes de formaldehído que en viviendas con menor número de fuentes $(\mathrm{RPC}=1.35 ; 95 \% \mathrm{IC}: 1.07-1.70)$. El resultado de la exposición al humo del cigarro no se modificó, viviendas con presencia de fumadores tuvieron menor probabilidad de presentar alta concentración de formaldehído $(\mathrm{RPC}=0.69 ; 95 \%$ IC: 0.52-0.90, Cuadro VI).

\section{DISCUSIÓN}

La concentración de formaldehído en las viviendas en Salvador fue menor que la reportada por otros estudios similares (Zhang et al. 1994, Báez et al. 2003, Gilbert et al. 2006, Guo et al. 2009). Las viviendas con una mejor ventilación y con un número mayor de fuentes de formaldehído presentaron las mayores concentraciones. Por otro lado, la presencia de fumadores dentro de la casa estuvo asociada a una menor concentración.

En Brasil se ha recomendado $100 \mu \mathrm{g} / \mathrm{m}^{3}$ de formaldehído como nivel máximo para la concentración en el aire interior (Gioda y Aquino 2003). Ese límite corresponde a una adaptación de los niveles máximos determinados internacionalmente en el contexto brasileño, tomando como referencia las recomendaciones de organizaciones internacionales y nacionales. La Organización Mundial de la Salud (OMS) recomienda que la concentración en ambientes interiores no industriales, no exceda los $100 \mu \mathrm{g} / \mathrm{m}^{3}$ en $30 \mathrm{~min}$ de exposición para individuos con buena salud y $10 \mu \mathrm{g} / \mathrm{m}^{3}$ para personas con hipersensibilidad respiratoria (González-Flesca et al. 1999). En las viviendas en Salvador la concentración de formaldehído fue inferior al límite nacional sugerido.

En varios países la legislación nacional define entre 100 a $120 \mu \mathrm{g} / \mathrm{m}^{3}$ de formaldehído como valor máximo permisible en el interior de domicilios. La mayoría de los estudios han reportado concentraciones por abajo del límite máximo permisible (Cuadro VII). Las principales fuentes de formaldehído se encuentran

CUADRO V. PROPORCIÓN DE VIVENDAS CON CONCENTRACIÓN DE FORMALDEHÍDO SUPERIOR A LA MEDIANA SEGÚN REAGRUPAMIENTO DE LAS CATEGORÍAS DE VENTILACIÓN Y FUENTES DEL FORMALDEHIDO

\begin{tabular}{lcc}
\hline $\begin{array}{l}\text { Indicadores del análisis de } \\
\text { conglomerados }\end{array}$ & $\begin{array}{l}\text { Proporción de viviendas con mayor } \\
\text { concentración de formaldehído (\%) }\end{array}$ & p-valor* \\
\hline $\begin{array}{l}\text { Grupos de ventilación } \\
\text { Poca-moderada ventilación }(\mathrm{n}=682)\end{array}$ & 47.2 & $\mathbf{0 . 0 1 5}$ \\
$\begin{array}{l}\text { Buena ventilación }(\mathrm{n}=178) \\
\text { Grupos de fuentes de formaldehído }\end{array}$ & 54.5 & \\
$\begin{array}{l}\text { Pocas fuentes }(\mathrm{n}=629) \\
\text { Moderada-múltiples fuentes }(\mathrm{n}=391)\end{array}$ & 46.6 & $\mathbf{0 . 0 0 7}$ \\
\hline
\end{tabular}

* prueba $\chi^{2}$ de asociación, números en negritas denotan diferencias significativas 
CUADRO VI. CÁLCULO DE LARAZÓN DE PRODUCTOS CRUZADOS (RPC) Y SUS INTERVALOS DE 95\% DE CONFIANZA SEGÚN MODELO DE REGRESIÓN LOGÍSTICA

\begin{tabular}{lcc}
\hline Variables & RPC & $95 \%$ IC** \\
\hline $\begin{array}{l}\text { Buena ventilación } \\
\quad(\text { Ref = poca-moderada ventilación) }\end{array}$ & $\mathbf{1 . 3 0}$ & $\mathbf{( 1 . 0 3 ; ~ 1 . 6 5 )}$ \\
$\begin{array}{l}\text { Moderadas-múltiples fuentes de } \\
\text { formaldehído } \\
\quad(\text { Ref }=\text { pocas fuentes })\end{array}$ & $\mathbf{1 . 3 5}$ & $\mathbf{( 1 . 0 7 ; ~ 1 . 7 0 )}$ \\
$\begin{array}{l}\text { Presencia de fumadores en la vivienda } \\
\quad(\text { Ref }=\text { ausencia de fumadores) }\end{array}$ & $\mathbf{0 . 6 9}$ & $\mathbf{( 0 . 5 2 ; 0 . 9 0 )}$ \\
\hline
\end{tabular}

Ref $=$ grupo de referencia, $\mathrm{IC}=$ Intervalo de confianza, números en negritas denotan diferencias significativas

en los ambientes interiores (González-Flesca et al. 1999, Clarisse et al. 2003, Gilbert et al. 2006, Ohura et al. 2006, de Bruin et al. 2008), por eso una buena ventilación disminuiría la concentración. Si por el contrario, la concentración en la atmósfera fuera mayor, ésta aumentaría en el interior. En nuestro estudio la ventilación se evaluó de forma indirecta, a través de la medición del área de ventanas, puertas y espacios abiertos dentro de la vivienda, se identificó el periodo de tiempo que las ventanas permanecían abiertas y se registró durante la toma de información, la percepción de olores fuertes dentro de la casa, lo que podría significar poca circulación del aire.

Una posible hipótesis a ser estudiada para explicar el resultado de la relación inversa entre la ventilación y la concentración de formaldehído en esta investigación, además de una posible fuente externa, es una relación indirecta entre mejor ventilación y mejor nivel socio-económico. Dicho nivel determinaría el tipo de muebles y la frecuencia de las remodelaciones en las viviendas, que aportarían una mayor contribución de fuentes emisoras de formaldehído en el interior de los hogares.

No fue objeto de este estudio medir la concentración de formaldehído en el exterior de las viviendas, sin embargo, habría sido útil identificar fuentes exteriores para obtener una posible explicación de los resultados. En Salvador se tiene como referencia de concentraciones en el aire exterior un rango de entre $0.24 \mu \mathrm{g}$ a $108.24 \mu \mathrm{g}$, provenientes principalmente de la emisión de gases del combustible vehicular (de Andrade et al. 1998). La emisión de gases por los vehículos podría ser una posible fuente externa en vista que no existen polos industriales o petroquímicos dentro del perímetro de la ciudad. En Brasil desde 1979 se utiliza metanol y etanol hidratado

CUADRO VII. CONCENTRACIÓN DE FORMALDEHÍDO EN EL AIRE INTERIOR DE VIVIENDAS Y LÍMITES ESTABLECIDOS PARA AMBIENTES NO INDUSTRIALES EN OTROS ESTUDIOS

\begin{tabular}{lccl}
\hline Lugar & $\begin{array}{c}\text { Concentración media } \\
\left(\mu \mathrm{g} / \mathrm{m}^{3}\right)\end{array}$ & $\begin{array}{c}\text { Límite de tolerancia } \\
\left(\mu \mathrm{g} / \mathrm{m}^{3}\right)\end{array}$ & Referencia \\
\hline Río de Janeiro, Brasil & 40 & 100 & Gioda y Aquino 2003 \\
San Pablo, Brasil & 35.5 & “ & Pires y Carvalho 2008 \\
Hong Kong, China & $<70$ & 125 & Lee et al. 1999 \\
Perth, Australia & 27.9 & Dingle y Frankling 2002 \\
Victoria, Australia & 15.8 & 120 & Garret et al. 1997 \\
Prince Edward Island, Canadá & 33.2 & Gilbert et al. 2005 \\
Quebec, Canadá & 29.5 & 100 & Gilbert et al. 2006 \\
Nueva York / Los Ángeles, E. U. & $17.7 / 19.3$ & “ & Sax et al. 2006 \\
Shimizu, Japón & 15.4 & 120 & Gura et al. 2006 al. 2009 \\
Hong Kong, China & 112.3 & & Heroux et al. 2010 \\
Regina, Canadá & $31.1(\mathrm{verano})$ & 135 & Dally et al. 1981 \\
& $23.4(\mathrm{invierno})$ & 122 & Zhang et al. 1994 \\
Wisconsin, Estados Unidos & 430 & 100 & Venn et al. 2003 \\
Nueva Jersey, E. U. & 66.7 & 125 & Vaizoglu et al. 2003 \\
Nottingham, Reino Unido & 22 & 80 & Zhao et al. 2004 \\
Ankara, Turquía & 80 & “ & Zhai et al. 2013 \\
Dalian, China & 130 & 100 & Clarisse et al. 2003 \\
Shenyang, China & 75 & “ & Marchand et al. 2008 \\
París, Francia & 34.4 & “ & Sakai et al. 2004 \\
Strasbourg, França & 33.5 & González-Flesca \\
Nagoya, Japón & 17.6 & Esta investigación \\
Nancy, Francia & 25 & 0.8 & \\
Salvador, Brasil & & & \\
\hline & & &
\end{tabular}


como combustible vehicular, éstos emiten más aldehídos que la gasolina o el diésel. Con el método de muestreo utilizado en este trabajo el acetaldehído no interfirió en la cuantificación del formaldehído.

Otros estudios en Brasil han mostrado resultados similares a los nuestros, mayores concentraciones en ambientes interiores donde se hacía uso de la ventilación natural (Pires y Carvalho 1999, Gioda y Aquino 2003). En México lugares con ventilación natural mostraron altas concentraciones interiores. Hubo correlación positiva entre las concentraciones interiores y las exteriores, atribuyéndosele a la emisión vehicular y a las diferentes condiciones del clima (Báez et al. 2003). En Salvador las condiciones del clima son relativamente estables durante todo el año por lo que todos los filtros fueron expuestos a condiciones similares.

En estudios internacionales la calidad de la ventilación se ha evaluado tomando en cuenta la ventilación natural (Báez et al. 2003, Zhao et al. 2004) y los aparatos para dicha ventilación (Vaizoglu et al. 2003, Park e Ikeda 2006). En países fríos han medido la concentración de dióxido de carbono como indicador de una ventilación deficiente (Dally et al. 1981), o utilizado gas traza para medir la tasa de renovación de aire/hora (Gilbert et al. 2006).

Resultados de la evaluación del efecto de la ventilación natural sobre la concentración de formaldehído pueden ser diferentes e incluso controversiales para una misma localidad y vivienda (Vaizoglu et al. 2003). La mayoría han reportado asociación entre una mejor ventilación y una menor concentración (Zhao et al. 2004, Gilbert et al. 2006, Heroux et al. 2010, Hullin et al. 2010, Brown et al. 2015). Sin embargo, ha sido reportada una mayor concentración ante mejores condiciones de ventilación (Pires y Carvalho 1999, Báez et al. 2003) y pocos han mostrado que sin importar el tipo de ventilación que se utilice, la concentración va a estar determinada por otros factores como la presencia de fuentes interiores y la antigüedad del inmueble (Hun et al. 2010).

En este estudio los muebles aparentaban tener mucho tiempo de uso, inclusive algunos estaban seriamente dañados. Sin embargo, se identificó una mayor concentración de formaldehído en viviendas con muebles de madera maciza, aglomerada o contrachapada (plywood, término en inglés). Se espera que la emisión de formaldehído disminuya conforme pasa el tiempo (Kim y Kim 2005), sin embargo la madera es una de sus principales fuentes de emisión en interiores.

En San Pablo, la presencia de muebles de madera estuvo asociada a altas concentraciones de formaldehído en interiores (Pires y Carvalho 1999). Los muebles hechos a base de aglomerados de fibra de madera o madera contrachapada aportan una mayor emisión de formaldehído en comparación con otro tipo de materiales, probablemente debido al uso de pegamento de urea-formaldehído (Garret et al. 1997, Zhao et al. 2004, Park e Ikeda 2006, Sax et al. 2006,). Entre menor el tiempo de antigüedad de los muebles mayor será la emisión de formaldehído (Gilbert et al. 2006). Bajas concentraciones se han encontrado en viviendas donde no ha habido muebles nuevos (Lee et al. 1999).

La población humana analizada en este trabajo, vivía predominantemente en casas independientes $(69.3 \%)$, localizadas en el nivel de terreno, sin espacio entre una casa y otra. Algunas familias vivían en apartamentos en pisos superiores al nivel del terreno. Las viviendas que pasaron por remodelación o pintura en los últimos seis meses presentaron mayor concentración de formaldehído. En Salvador, las viviendas se construyen con bloques cerámicos de barro, madera (o productos de madera aglomerada), ladrillos y cerámicas para pisos, además, las pinturas que se utilizan son predominantemente a base de agua. La madera y las pinturas son las que probablemente contribuyeron más en la concentración de formaldehído.

Las pinturas tienen en su composición resinas, clorato de plata y bióxido de titanio. Las resinas naturales son sustancias extraídas de la madera de los árboles, las sintéticas pasan por un proceso de polimerización siendo ampliamente utilizadas en la fabricación de pinturas y adhesivos. La resina acrílica y la vinílica son utilizadas en la composición de pinturas de uso arquitectónico.

En estudios internacionales se ha comprobado que viviendas en donde las paredes habían sido remodeladas o pintadas, o el piso renovado durante los últimos seis meses, presentaban una mayor concentración de formaldehído interior (Lee et al. 1999, Clarisse et al. 2003, Gilbert et al. 2006, Park e Ikeda 2006). El uso de pintura para madera libera una mayor concentración en comparación a otro tipo de pintura (Weislander et al. 1997). Sin embargo, la asociación entre pintura reciente y altas concentraciones de formaldehído no ha sido relevante en todos los estudios (Gilbert et al. 2005, Marchand et al. 2008).

La ventilación deficiente y la emisión de gases por la madera han sido identificados como "factores de confusión", que aumentan o disminuyen la fuerza de la asociación entre la antigüedad de la vivienda y la concentración de formaldehído (Gilbert et al. 2005). En nuestro estudio la 
antigüedad mayor a cinco años no estuvo asociada con la concentración de formaldehído. Entre menor tiempo de antigüedad, mayor será la concentración debido al material de construcción, la pintura y los muebles nuevos. Viviendas con una antigüedad menor de un año han registrado las concentraciones más altas (Zhang et al. 1994, Dingle y Frankling 2002, Zhao et al. 2004, Ohura et al. 2006, Guo et al. 2009), y pueden continuar con dichos niveles hasta cinco o siete años después de haber sido construidas (Sakai et al. 2004, Gilbert et al. 2005, Heroux et al. 2010). Una antigüedad mayor a diez años no siempre se ha asociado significativamente con la concentración interior (Vaizoglu et al. 2003, Gilbert et al. 2006, Marchand et al. 2008, Hun et al. 2010, Brown et al. 2015).

La utilización de más de siete productos de limpieza dentro de la vivienda aumentó la concentración de formaldehído en Salvador. Los productos de limpieza constituyen una mezcla de múltiples compuestos químicos con diferentes grados de volatilidad y diversas propiedades químicas, capaces de reaccionar con otros contaminantes del aire para generar otros compuestos potencialmente peligrosos. La tasa de emisión de los compuestos primarios va a depender de la composición del producto, la concentración de componentes volátiles en el producto, el patrón de uso (cantidad y frecuencia), la forma de aplicación y las características físicas y químicas de los compuestos (Nazaroff y Weschler 2004).

En Brasil esos productos son agrupados y clasificados dependiendo de la función técnica que desempeñan y el propósito de su uso. La agencia nacional de vigilancia de la salud (ANVISA) clasifica dentro de este grupo a los detergentes y sus congéneres: blanqueadores, desinfectantes, desodorantes, esterilizantes, alguicidas, fungicidas, desinfectante de agua para consumo humano, cloro, productos biológicos, insecticidas, raticidas, productos para jardinería y repelentes (ANVISA 2001).

La población humana en este trabajo, hacía uso principalmente de blanqueadores (96.9\%), desinfectantes $(92.8 \%)$ y jabones $(76.5 \%)$. Los blanqueadores $(84.3 \%)$ y los jabones $(93.3 \%)$ eran de origen industrial, mientras que los desinfectantes eran fabricados artesanalmente $(50.3 \%)$. Los jabones $(97.1 \%)$ y los desinfectantes $(65.7 \%)$ se usaban diariamente en la mayor parte de las viviendas, en tanto que los blanqueadores fueron de uso frecuente (3-6 veces/semana) en $27.7 \%$ de las casas.

El formaldehído actúa como compuesto activo o como conservador de cosméticos y de otros productos de uso diario (Zock 2005). Se identificó como el conservador más común en productos de limpieza en Dinamarca (Flyvholm 2005), pero en Massachusetts, Estados Unidos, raramente formaba parte de productos de limpieza en hospitales (Bello et al. 2009). En Minas Gerais, Brasil, el formaldehído fue identificado como principio activo de desinfectantes de fabricación artesanal (de Oliveira et al. 2012). En San Pablo, su concentración fue alta en un hospital $\left(210 \mu \mathrm{g} / \mathrm{m}^{3}\right)$ (Pires y Carvalho 2008).

La presencia de fumadores en las viviendas en Salvador estuvo asociada a una menor concentración de formaldehído. La prevalencia de tabaquismo en nuestro estudio fue de $24.3 \%$ entre los padres o familiares quienes consumían cigarros fabricados industrialmente $(90.7 \%)$, en promedio 15 unidades por persona/día. El $54.7 \%$ de los fumadores informaron que lo hacían en lugares fuera de la vivienda como patios, aceras, o espacios laterales. Después de ajustar por el efecto de otras fuentes de formaldehído y de la ventilación, la asociación entre el humo del cigarro y la concentración de formaldehído se mantuvo. Estudios nacionales han reportado mayores concentraciones de formaldehído $\left(105 \mu \mathrm{g} / \mathrm{m}^{3}\right)$ en locales sin fumadores (Pires y Carvalho 1999) y no han mostrado una correlación significativa entre la concentración de nicotina (derivada del humo del cigarro) y la concentración de formaldehído (Brickus et al. 1998).

En estudios internacionales la concentración interior ha sido menor en presencia de fumadores $\left(<50 \mu \mathrm{g} / \mathrm{m}^{3}\right)$ (Lee et al. 1999, Dingle y Frankling 2002), o no ha habido asociación entre la presencia de humo del cigarro en el ambiente y la concentración interior (Weislander et al. 1997, Clarisse et al. 2003, Vaizoglu et al. 2003, Gilbert et al. 2005, Gilbert et al. 2006, Guo et al. 2009, Heroux et al. 2010, Brown et al. 2015). Sin embargo, otros han atribuido la concentración de formaldehído interior a la presencia de fumadores (Báez et al. 2003, Villanueva et al. 2015), o al número de cigarrillos fumados dentro del ambiente, sobre todo durante el invierno (Ohura et al. 2006).

Los estudios experimentales han reportado concentraciones altas de formaldehído siendo emitidas en el humo del cigarrillo (10 a > $100 \mu \mathrm{g} /$ cigarrillo), pero han sido considerados de limitada utilidad en la evaluación del efecto del humo del cigarro sobre la concentración de formaldehído interior, debido a que ese tipo de estudios no han utilizado un método estándar para medirlo y han reportado alta variabilidad en la concentración del formaldehído resultante. Se considera que aún en circunstancias extremas el efecto del humo del cigarro sobre los 
niveles de formaldehído podría no ser determinante (Godish 1999). Debido a eso sería necesario medir la concentración durante y posteriormente al uso de cigarros, para determinar si el efecto es transitorio o residual (Dally et al. 1981).

Una posible limitación de este estudio se refiere al hecho de que la información sobre los factores de exposición fue reportada por los padres o responsables de los niños, lo que podría haber introducido un sesgo en la información. Sin embargo, algunas recomendaciones fueron tomadas en cuenta durante el diseño y aplicación del cuestionario para minimizar ese posible error. Medir la concentración de formaldehído en el aire exterior de las casas probablemente habría facilitado la posibilidad de inferir sobre la relación entre la concentración interior y la exterior, pero no fue objeto de esta investigación.

\section{CONCLUSIONES}

La concentración de formaldehído en el interior de las viviendas en Salvador fue menor que los niveles reportados por otros estudios nacionales e internacionales. Mejores condiciones de ventilación natural y la presencia de un mayor número de fuentes potencialmente emisoras aumentaron la concentración. En contraste, la presencia del humo del cigarro en el ambiente la disminuyó. El método de muestreo ambiental utilizado, permitió la determinación de la presencia del formaldehído en el aire con suficiente confiabilidad.

Los resultados han permitido conocer la concentración de formaldehído que cotidianamente se produce en el interior de viviendas convencionales en Salvador, así como algunos factores del ambiente que muestran que posiblemente la contribución de las fuentes exteriores sea tan importante como la de las fuentes interiores para dicha concentración. El conocimiento sobre el nivel de exposición podrá ayudar en la evaluación del riesgo a la salud de los habitantes en esos ambientes y en la definición de los parámetros mínimos para una buena calidad del aire en interiores. Medidas de prevención tales como el control de la concentración del formaldehído en los materiales de construcción, en la confección de muebles para el hogar y en la fabricación de productos de limpieza, deberán ser planificadas. Los datos de esta investigación podrían ser utilizados en la determinación de la concentración estándar de formaldehído en otros países en desarrollo con condiciones del clima, economía y cultura similares a los de la población de Salvador, Brasil.

\section{AGRADECIMIENTOS}

Este estudio recibió financiamiento de The Wellcom Trust del Reino Unido, a través del programa Major Awards to Centres of Excellence in Latin America. El Conselho Nacional de Desenvolvimento Tecnológico (CNPq) de Brasil financió la beca de doctorado.

Artículo basado en la tesis de doctorado de Norma Irene Soza Pineda, presentada al Programa de Post-Graduación en Salud Colectiva, Universidad Federal de Bahía, Brasil, 2009.

\section{REFERENCIAS}

ANVISA (2001). Resolução da Diretoria Colegiada - RDC $\mathrm{n}^{\circ}$ 184. Agencia Nacional de Vigilância Sanitária. Brasil. 22 de octubre de 2001.

Báez A., Padilla H., García R., Torrez M.C., Rosas I. y Belmont R.D. (2003). Carbonyl levels in indoor and outdoor air in México City and Xalapa, México. Sci. Total. Environ. 302 (1-3), 211-226. DOI: $10.1016 / \mathrm{S} 0048-9697(02) 00344-3$

Báez A.P., Padilla H.G., García R.M., Belmont R.D. y Torrez M.C. (2006). Measurement of indoor-outdoor carbonyls at four residential homes in Mexico City metropolitan area. Int. J. Environ. Pollution 26 (1-2-3), 90-105. DOI: 10.1504/IJEP.2006.009100

Barreto M.L., Cunha S.S., Alcântara-Neves N., Carvalho L.P., Cruz A.A., Stein R., Genser B. Cooper P.J.y Rodrigues L.C. (2006). Risk factors and immunological pathways for asthma and other allergic diseases in children: background and methodology of a longitudinal study in a large urban center in Northeastern Brazil (Salvador-SCAALA study). BMC. Pulm. Med. 6, 6-15.

DOI: $10.1186 / 1471-2466-6-15$

Bello A., Quinn M.M., Perry M.J. y Milton D.K. (2009). Characterization of occupational exposures to cleaning products used for common cleaning tasks-a pilot study of hospital cleaners. Environ. Health 8, 1-11. DOI: 10.1186/1476-069X-8-11

Brickus L.S.R., Cardoso J.N. y de Aquino Neto F.N. (1998). Distributions of indoor and outdoor air pollutants in Rio de Janeiro, Brazil: implications to indoor air quality and bayside offices. Environ. Sci. Technol. 32 (22), 3485-3490. DOI: 10.1021/es980336x

Brown T., Dassonville C., Derbez M., Ramalho O., Kirchner S., Crump D. y Mandin C. (2015). Relationships between socioeconomic and lifestyle factors and indoor air quality in French dwellings. Environ. Res. 140, 385-396. DOI: 10.1016/j.envres.2015.04.012 
Clarisse B., Laurent A.M., Seta N., Le Moullec Y., El Hasnaoui A. y Momas I. (2003). Indoor aldehydes: measurement of contamination levels and identification of their determinants in Paris dwellings. Environ. Res. 92 (3), 245-253. DOI: 10.1016/S0013-9351(03)00039-2

Corrêa S.M. y Arbilla G. (2005). Formaldehyde and acetaldehyde associated with the use of natural gas as a fuel for light vehicles. Atmos. Environ. 39 (25), 4513-4518. DOI: 10.1016/j.atmosenv.2005.03.042

De Bruin Y.B., Koistinen K., Kephalopoulus S., Geiss O., Tirendi S. y Kotzias D. (2008). Characterisation of urban inhalation exposures to benzene, formaldehyde and acetaldehyde in European Union. Environ. Sci. Pollut. Res. 15 (5), 417-430.

DOI: 10.1007/s11356-008-0013-4

de Andrade J.B., Pinheiro H.L.C. y de Andrade M.V. (1995). The formaldehyde and acetaldehyde content of atmospheric aerosol. J. Bras. Chem. Soc. 6 (3), 287290. DOI: $10.5935 / 0103-5053.19950048$

de Andrade J.B., de Andrade M.V. y Pinheiro H.L. C. (1998). Atmospheric levels of formaldehyde and acetaldehyde and their relationship with the vehicular fleet composition in Salvador, Bahia, Brazil. J. Bras. Chem. Soc. 9 (3), 219-223.

DOI: $10.1590 / \mathrm{S} 0103-50531998000300004$

de Andrade M.V.A.S., Pinheiro H.L.C., Pereira P.A.P. y de Andrade J.B. (2002). Compostos carbonílicos atmosféricos: fontes, reatividade, níveis de concentração e efeitos toxicológicos. Quim. Nova 25 (6b), 1117-1131. DOI: 10.1590/S0100-40422002000700013

de Oliveira V.L.S, Caetano R.M., y Gomes F.C.O. (2012) Avaliação da qualidade de saneantes clandestinos comercializados em Belo Horizonte, Minas Gerais. Rev. Ciên. Farm. Básica Apl. 33 (4), 77-582.

Daily K.A., Hanrahan L.P., Woodbury M.A. y Kanarek M.S. (1981). Formaldehyde exposure in nonoccupational environment. Arch. Environ. Health 36 (6), 277-284. DOI: 10.1080/00039896.1981.10667638

Dingle P. y Frankling P. (2002). Formaldehyde levels and the factors affecting these levels in homes in Perth, Western Australia. Indoor Built. Environ. 11, 111-116. DOI: $10.1177 / 1420326$ X0201100206

Flyvholm M.A. (2005) Preservatives in registered chemical products. Contact. Dermatitis 53 (1), 27-32. DOI: $10.1111 / \mathrm{j} .0105-1873.2005 .00629 . \mathrm{x}$

Garret M.H., Hooper M.A. y Hooper B.M. (1997). Formaldehyde in Australian homes: levels and sources. Clean Air. Environ. Quality 31 (2), 28-32.

Garret M.H., Hooper M.A., Rayment P.R. y Abramson M.J. (1999). Increased risk of allergy in children due to formaldehyde exposure in home. Allergy 54 (4), 330-337.

DOI: $10.1034 / j .1398-9995.1999 .00763 . x$
Gilbert N.L., Guay M., Miller D., Judek S., Chan C.C. y Dales R.E. (2005). Levels and determinants of formaldehyde, acetaldehyde and acrolein in residential indoor air in Prince Edward Island Canada. Environ. Res. 99 (1), 11-17. DOI: 10.1016/j.envres.2004.09.009

Gilbert N.L., Gauvin D., Guay M., Héroux M.-E., Dupuis G., Legris M., Chan C.C., Dietz R.N. y Lévesque B. (2006). Housing characteristics and indoor concentrations of nitrogen dioxide and formaldehyde in Quebec City, Canada. Environ. Res. 102 (1), 1-8.

DOI: 10.1016/j.envres.2006.02.007

Gioda A. y Aquino Neto F.R. (2003). Poluição química relacionado ao ar de interiores no Brasil. Quim. Nova 26 (3), 359-365.

DOI: $10.1590 / \mathrm{S} 0100-40422003000300013$

Godish T. (1989). Formaldehyde exposures from tobacco smoke: a review. Am. J. Public Health 79 (8), 10441045. DOI: 10.2105/AJPH.79.8.1044

Gonzalez-Flesca N., Cicolella A., Bates M. y Bastin E. (1999) Pilot study of personal, indoor and outdoor exposure to benzene, formaldehyde and acetaldehyde. Environ. Sci. Pollut. Res. 6 (2), 95-102.

DOI: $10.1007 / \mathrm{BF} 02987560$

Guo H., Kwok N.H., Cheng H.R., Lee S.C., Hung W.T. y Li Y.S. (2009). Formaldehyde and organic volatile compounds in Hong Kong homes: concentrations and impact factors. Indoor Air 19 (3), 206-217.

DOI: 10.1111/j.1600-0668.2008.00580.x

Heroux M.E., Clark N., Ryswyk K.V., Mallick R., Gilbert N.L., Harrison I., Rispler K., Wang D., Anastassopoulos A., Guay M., MacNeill M. y Wheeler A.J. (2010). Predictors on indoor air concentration in smoking and non-smoking residence. Int. J. Environ. Res. Public Health 7 (8), 3080-3099.

DOI: $10.3390 /$ ijerph7083080

Hulin M., Caillaud D. y Annesi-Maesano I. (2010). Indoor air pollution and childhood asthma: variations between urban and rural areas. Indoor Air 20 (6), 502-514. DOI: 10.1111/j.1600-0668.2010.00673.x

Hun D.E., Corsi R.L., Morandi M.T. y Siegel J.A. (2010). Formaldehyde in residences: long-term indoor concentrations and influencing factors. Indoor Air 20 (3), 196-203. DOI: 10.1111/j.1600-0668.2010.00644.x

IBGE (2000). Censo brasileiro 2000. Instituto brasileiro de geografia e estatística [en línea]. www.ibge.gov.br/ home/estatistica/populacao/contagem2007/contagem. pdf 20/10/2009

IOM (2004). Clearing the air: Asthma and indoor air exposures. Institute of medicine. National Academy Press. Washington, EUA, 438 pp.

Kelly T.J., Smith D.L. y Satola J. (1999). Emission rates of formaldehyde from materials and consumer products 
forms in California homes. Environ. Sci. Technol. 33 (1), 81-88. DOI: 10.1021/es980592

Kim S. y Kim H.J. (2005). Comparison of formaldehyde emission from building finishing materials at various temperatures in under heating system; ONDOL. Indoor Air 15 (5), 317-325. DOI: $10.1111 /$ j.1600-0668.2005.00368.x

Klepeis N.E., Nelson W.C., Ott W.R., Robinson J.P., Tsang A.M., Switzer P., Behar J.V., Hern S.C. y Engelmann W.H. (2001). The national human activity patterns survey (NHAPS): a resource for assessing exposure to environmental pollutants. J. Expos. Anal. Environ. Epidemiol. 11 (3), 231-252. DOI: 10.1038/sj.jea.7500165

Lee S.C., Chang M. y Chan K.Y. (1999). Indoor and outdoor air quality investigation at six residential buildings in Hong Kong. Environ. Int. 25 (4), 489-496. DOI: 10.1016/S0160-4120(99)00014-8

Levin J., Andersson K., Linndahl R. y Nilsson C. (1985). Determination of sub-parts-per-million levels of formaldehyde in air using active and passive sampling on 2,4-dinitrophenylhydrazine-coated glass fibre and high-performance liquid chromatography. Anal. Chem. 57 (6), 1032-1035.

DOI: $10.1021 / \mathrm{ac} 00283 \mathrm{a} 016$

Marchand C., Le Calvé S., Mirabel Ph., Glasser N., Casset A., Schneider N. y de Blay F. (2008). Concentrations and determinants of gaseous aldehydes in 162 homes in Strasbourg (France). Atmos. Environ. 42 (3), 505-516. DOI: 10.1016/j.atmosenv.2007.09.054

Nazaroff W.W. y Weschler C.J. (2004). Cleaning products anda ir fresheners: exposure to primary and secondary pollutants. Atmos. Environ. 38 (18), 2841-2865. DOI: 10.1016/j.atmosenv.2004.02.040

Norbäck D., Björnsson E., Janson C., Widstrom J. y Boman G. (1995) Asthmatic symptoms and volatile organic compounds, formaldehyde, and carbon dioxide in dwellings. Occup. Environ. Med. 52, 388-395. DOI: $10.1136 /$ oem.52.6.388

Ohura T., Amagai T., Senga Y. y Fusaya M. (2006). Organic air pollutants inside and outside residences in Shimizu, Japan: levels, source and risk. Sci. Total. Environ. 366 (2-3), 485-499.

DOI: $10.1016 /$ j.scitotenv.2005.10.005

Park J.S. y Ikeda K. (2006). Variations and formaldehyde and VOCS levels during 3 years and new and older homes. Indoor Air 16 (2), 129-135. DOI: 10.1111/j.1600-0668.2005.00408.x

Pinheiro H.L.C., de Andrade M.V., Pereira P.P.A. y de Andrade J.B. (2004). Spectrofluorimetric determination of formaldehyde in air after collection onto silica cartridges coated with Fluoral P. Microchem. J. 78 (1), 15-20.

DOI: $10.1016 /$ j.microc.2004.02.017
Pires M. y Carvalho L.R.F. (1999). Presença de compostos carbonílicos no ar em ambientes internos na cidade de São Paulo. Quim. Nova 22 (4), 487-496. DOI: $10.1590 /$ S0100-40421999000400004

Runchev K.B., Spickett J.T., Bulsara M.K., Phillips M.R. y Stick S.M. (2002). Domestic exposure to formaldehyde significantly increases the risk of asthma in young children. Eur. Respir. J. 20, 403-408.

DOI: 10.1183/09031936.02.00245002

Sakai K., Norbäck D., Mi Y., Shibata E., Kamijima M., Yamada T. y Takeuchi Y. (2004). A comparison of indoor air pollutants in Japan and Sweden: formaldehyde, nitrogen dioxide, and chlorinated volatile organic compounds. Environ. Res. 94 (1), 75-85.

DOI: 10.1016/S0013-9351(03)00140-3

Salthammer T., Mentese S. y Marutzky R. (2010). Formaldehyde in the indoor environment. Chem. Rev. 110 (4), 2536-2572. DOI: 10.1021/cr800399g

Sax S., Bennett D.H., Chillrud S.N., Ross J., Kinney P.L. y Spengler J.D. (2006) A cancer risk assessment of inner-city teenagers living in New York city and Los Angeles. Environ. Health Perspect. 114 (10), 15581566. DOI: 10.1289/ehp.8507

Vaizoglu S.A., Aycan S., Deveci M.A., Acer T., Bulut B., Bayraktar U.D., Akyollu B., Celik M., Arslan U., Akpinar F., Baris Z., Arslan S., Deniz A., Evci E. D. y Güller Ç. (2003). Determining domestic formaldehyde levels in Ankara, Turkey. Indoor Built Environ. 12 (5), 329-335. DOI: 10.1177/142032603035546

Venn A.J., Cooper M., Antoniak M., Laughlin C., Britton J. y Lewis S.A. (2003). Effects of volatile organic compounds, damp, and other environmental exposures in the home on wheezing illness in children. Thorax 58 (11), 955-960. DOI: 10.1136/thorax.58.11.955

Villanueva F., Tapia A., Amo-Salas M., Notario A., Cabañas B. y Martínez E. (2015). Levels and sources of volatile organic compounds including carbonyls in indoor air of homes of Puertollano, the most industrialized city in central Iberian Peninsula. Estimation of health risk. Int. J. Hygiene Environ. Health 218 (6), 522-534. DOI: 10.1016/j.ijheh.2015.05.004

Weislander G., Norbäck D., Björnsonn B., Janson C. y Boman G. (1997). Asthma and the indoor environment: the significance of emission of formaldehyde and volatile organic compounds from newly painted indoor surfaces. Int. Arch. Occup. Environ. Health 69 (2), 115-124. DOI: 10.1007/s004200050125

Wolkoff P., Schneider T., Kildeso J., Degerth R., Jaroszewski M. y Schunk H. (1998). Risk in cleaning: chemical and physical exposure. Sci. Total. Environ. 215 (1-2), 135-156. DOI: 10.1016/S0048-9697(98)00110-7

Zhang J., He Q. y Lloy P.J. (1994). Characteristics of aldehydes: concentrations, sources, and exposures for 
indoor and outdoor residential microenvironments.

Environ. Sci. Technol. 28 (1), 146-152.

DOI: $10.1021 / \mathrm{es} 00050 \mathrm{a} 020$

Zhao Y., Chen B., Guo Y., Peng F. y Zhao J. (2004). Indoor air environment of residential buildings in Dalian,
China. Energy Build 36 (12), 1235-1239.

DOI: $10.1016 /$ j.enbuild.2003.09.011

Zock J.P. (2005). World at work: Cleaners. Occup. Environ. Med. 62, 581-584.

DOI: $10.1136 / \mathrm{oem} .2004 .015032$ 